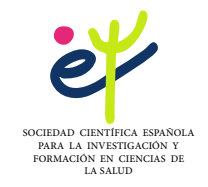

\title{
Desarrollo moral en contextos educativos: Una revisión sistemática
}

\section{Moral development in educational contexts: A systematic review}

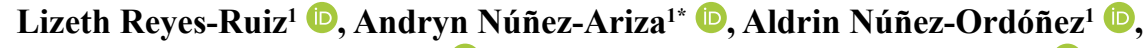 \\ Milgen Sánchez-Villegas $^{1}$ (D), Farid Alejandro Carmona Alvarado ${ }^{1}$ (D), \\ Patricia Unger Raphael Bataglia ${ }^{2}$ (i) \& Cristiane Paiva Alves ${ }^{3}$
}

${ }^{1}$ Universidad Simón Bolívar, Facultad de Ciencias Jurídicas y Sociales, Barranquilla, Colombia; ${ }^{2}$ Universidade Estadual Paulista, Departamento de Educação e Desenvolvimento Humano, Marília-SP, Brasil; ${ }^{3}$ Universidade Estadual Paulista, Departamento de Fisioterapia e Terapia Ocupacional, MaríliaSP, Brasil

\begin{abstract}
Resumen
El objetivo de esta revisión sistemática fue identificar la evidencia disponible en la literatura científica relacionadas con el estudio del desarrollo moral en contextos educativos. Se realizaron búsquedas en las bases de datos bibliográficas de PubMed, Scopus, Psychology and Behavioral Science Collection (EBSCO) y Scielo utilizando criterios de búsqueda predeterminados, siguiendo la metodología PRISMA. Se consideraron los estudios de investigación publicados desde 2010 hasta octubre de 2020 y, luego de la evaluación, fueron identificados 3.021 artículos que trataban el tema y se incluyeron finalmente 167 artículos en la revisión que cumplieron los criterios de inclusión. Los resultados sugieren la influencia del contexto educativo en el desarrollo moral, desde todos los niveles de formación educativa, donde convergen aspectos, individuales, cognitivos, socioeconómicos, interculturales, pedagógicos, institucionales, familiares e interactivos, incentivando al desarrollo de estudios que nutran la discusión científica sobre la evolución moral del ser humano y sus prácticas formativas asociadas.
\end{abstract}

Palabras clave: Desarrollo moral, contextos educativos, Educación

\footnotetext{
* Correspondencia: Andryn Núñez-Ariza, Universidad Simón Bolívar, Facultad de Ciencias Jurídicas y Sociales, Barranquilla, Colombia. Email: anunez10@unisimon.edu.co
} 


\begin{abstract}
The aim of this systematic review was to identify the evidence available in scientific literature in relation to moral development in educational contexts. The bibliographic databases of PubMed, Scopus, Psychology and Behavioral Science Collection (EBSCO) and Scielo were examined using predetermined search criteria and PRISMA methodology. Research studies published from 2010 to October 2020 were considered and, after evaluation, 3,021 articles dealing with the topic were identified and finally 167 articles were included in the review that met the inclusion criteria. The results suggest the influence of the educational context on moral development, from all levels of educational formation, where aspects, individual, cognitive, socioeconomic, intercultural, pedagogical, institutional, family and interactive converge, encouraging the development of studies that nourish the scientific discussion on the moral evolution of the human being and its associated training practices.
\end{abstract}

Keywords: Moral development; Educational contexts; Education

\title{
INTRODUCCIÓN
}

La moral ha sido relacionada con las acciones del ser humano, representado como un hombre libre, capaz de decidir a partir de su conocimiento y voluntad (Rodríguez, 2016); desde una perspectiva sociológica, la moral se genera a partir de fenómenos y hechos fundamentalmente sociales, ya que cada sociedad, en su desarrollo histórico, construye sus propias reglas morales, percibidas como sistemas, las que difieren de los sistemas morales de otros grupos sociales (Rohling, 2017, p.4). Por su parte, la postura cognitivo-evolutiva, se ha basado en la dimensión del conocimiento, destacando el desarrollo de las reglas y la adopción de principios universales, formulando una evolución progresiva de las estructuras morales del individuo — a medida que avanza su ciclo vital — forjado a partir de un orden moral externo; así, se ha conceptualizado el desarrollo moral, desde una visión global, considerando principios de justicia que generan el razonamiento del individuo, surgiendo juicios morales basados en nociones universales (Rodríguez, 2016).

En esta línea, según Kohlberg y Mayer (1972) la educación ejerce un papel fundamental en el proceso de desarrollo moral, desde la niñez hasta la edad adulta, ya que en este escenario se debe estimular al individuo en la interacción natural con su medio, buscando el desarrollo progresivo de su propia conciencia y juicio ante situaciones contextuales, constituyéndose como meta central propender al desarrollo, tanto intelectual como moral (Díaz-Serrano, 2015b; Kohlberg y Mayer, 1972).

Actualmente, la Organización de las Naciones Unidas para la Educación, la Ciencia y la Cultura, UNESCO (2020) ha planteado dentro de los objetivos de desarrollo sostenible ser 
garante de una educación inclusiva, equitativa y de calidad, a partir de la promoción de una educación que propenda al desarrollo de estilos de vida sostenibles, la adopción derechos humanos, la igualdad entre géneros, la promoción de una cultura de paz y no violencia, es decir, el fomento de una ciudadanía mundial.

A su vez, se ha considerado el desarrollo moral uno de los objetivos esenciales en los sistemas educativos, dada su progresión gradual, se requiere aunar esfuerzos que propendan a la evolución de competencias morales, articuladas con la formación académica y habilidades prácticas, destacando tres dimensiones esenciales, gestadas en escenarios de aprendizaje: transcición, reconstrucción e interiorización moral (Ranjbar et al., 2018).

Por su parte, revisiones previas han sugerido una erosión ética en la educación superior, con una tendencia a disminuir o mantenerse en sus estadios iniciales (Yuguero et al., 2019), de ahí que se deban sistematizar contribuciones investigativas sobre el desarrollo moral en escenarios formativos, considerando niveles de educación inicial, media y superior, para así conocer las dimensiones implicadas en la preparación del ser humano con la moral requerida para ser un ser social, promoviendo su juicio moral y la capacidad para tomar decisiones éticas (Kim y Park, 2019). Visto además como un eje transversal del aprendizaje, garante de una formación integral, encaminada al desarrollo de una sociedad democrática (Lagos-Vargas y Keupuchur-Natalini, 2019). Así, se evidencia el compromiso de los entes educativos en el desarrollo moral, social y emocional de los individuos, anudado al conocimiento técnico, posibilitando cambios y contribuciones a nivel comunitario y personal (Pedraza-Chávez y Pérez-Ramírez, 2019; Asif et al., 2020).

Ante este panorama, surgió el interés por revisar los hallazgos científicos asociados al desarrollo moral en contextos educativos, lo que llevó a realizar un análisis documental que involucró el rastreo, la sistematización y la revisión hermenéutica de artículos y estudios científicos divulgados con la comunidad académica, buscando generar un diálogo entre tendencias investigativas, puntos de encuentro y discrepancias, religando saberes mediante un proceso de identificación, categorización y comprensión, donde emergió una visión global e integradora de las variables de interés epistemológico (Londoño et al., 2014), que ha permitido dar respuesta al interrogante que movilizó el estudio, es decir, ¿Cuáles son los aportes investigativos asociados al desarrollo moral en contextos educativos?.

\section{MÉTODO}

\section{Procedimiento de búsqueda y parámetros de revisión}

Esta revisión sistemática aporta una síntesis y fundamentación sobre el desarrollo moral en contextos educativos. Ha posibilitado describir conceptualizaciones destacadas, consolidar hallazgos, identificar tendencias en metodologías y vacíos investigativos en el campo de interés (Baker, 2016). De este modo, se ha adoptado una metodología guiada 
por la diagramación PRISMA (Preferred Reporting Items for Systematic reviews and MetaAnayses) (Liberati et al., 2009), acogida por Okoli y Schabram (2010), buscando organizar y sistematizar las evidencias rastreadas, partiendo de los interrogantes que han orientado el estudio: ¿Cuáles son los aportes investigativos asociados al desarrollo moral en contextos educativos?, ¿Cuál ha sido la evidencia científica relacionada con el desarrollo moral, de acuerdo con los niveles de educación?.

Seguidamente, se realizó la búsqueda entre octubre y noviembre del 2020, en las bases de datos Scielo, PubMed, Scopus y EBSCO. Se hizo el rastreo sistemático haciendo uso de las combinaciones de descriptores "desarrollo moral AND educación” en español, y "moral development AND education" en inglés, estas ecuaciones definieron la búsqueda, a través de la herramienta Thesaurus. La revisión inicial se realizó solo de títulos y resúmenes o abstracts, arrojando un total de 3.065 artículos; sin embargo teniendo en cuenta los criterios de inclusión y exclusión, 167 artículos fueron seleccionados para su análisis a profundidad (ver gráfico 1), los cuales fueron sistematizados utilizando el programa de referencias bibliográficas ${ }^{\circledR}$ Mendely reference manager.

\section{Criterios de inclusión}

Durante el rastreo, fueron considerados artículos con población escolarizada, que mostraran la evidencia científica sobre su relación con el desarrollo moral, sin discriminar el modelo teórico y las técnicas implementadas; a su vez, fueron incluidos estudios de tipo cuantitativo, cualitativo y mixto, donde se divulgaran sus resultados investigativos, en el lapso comprendido entre los años 2010 y 2020. Finalmente, se seleccionaron artículos en inglés, portugués y español.

\section{Criterios de exclusión}

Se excluyeron artículos con poblaciones diferentes a sujetos escolarizados o fuera de contextos educativos. Además, debían ser estudios con variables o categorías asociadas al desarrollo moral. No se tuvieron en cuenta artículos de revisión sistemática, investigaciones documentales, metaanálsis, revisiones bibliográficas, tesis doctorales, o resultados parciales de estudios.

La selección de estos criterios posibilitó la depuración de los documentos publicados e identificados, excluyendo artículos con datos no relacionados con el objetivo del estudio; a continuación, en el Gráfico 1, ha sido representado el diagrama de flujo del proceso de rastreo y sistematización, guiado por las indicaciones PRISMA. 


\section{Gráfico 1.}

Diagrama PRISMA. Fuente: elaboración propia.

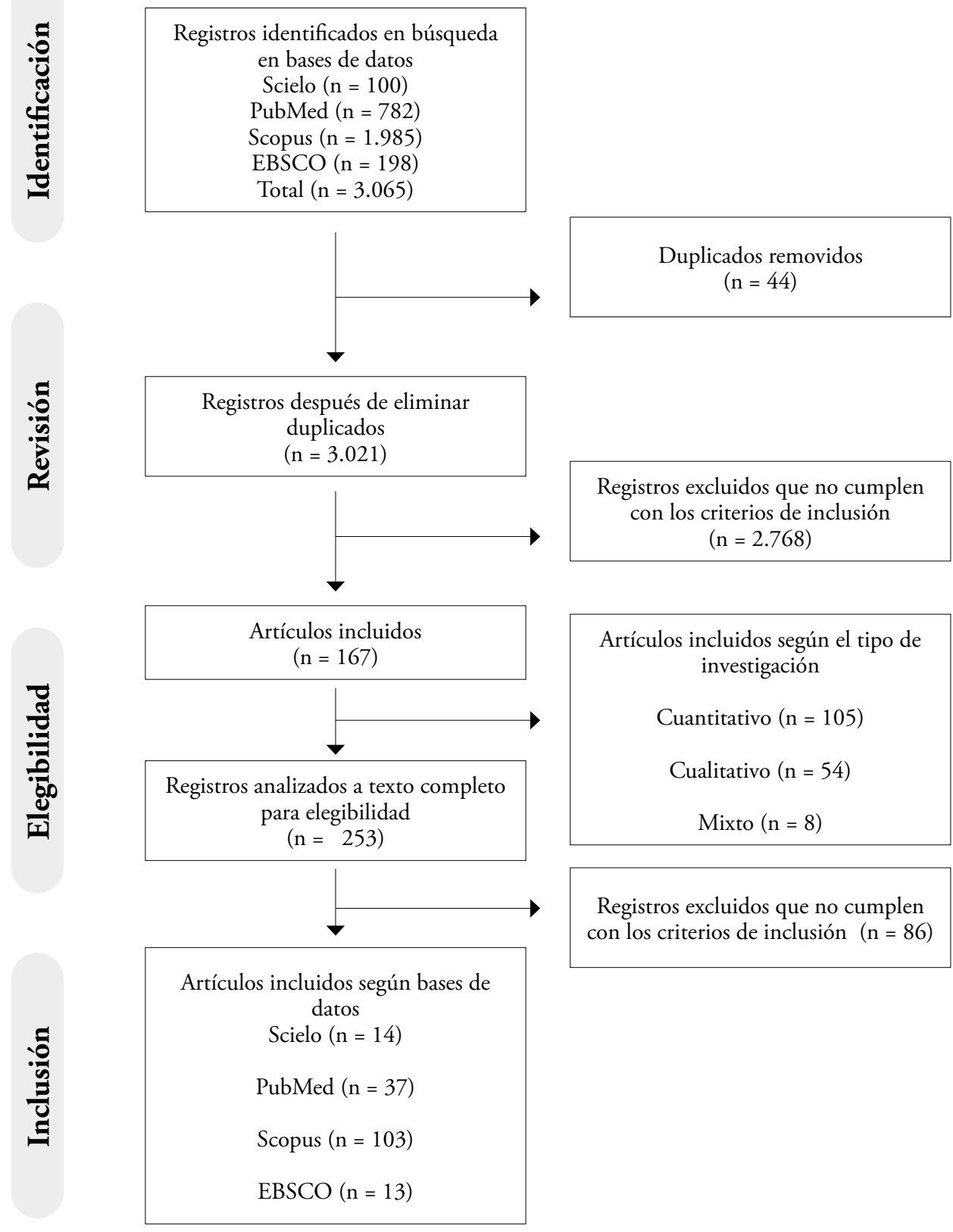




\section{RESULTADOS}

A partir de la revisión sistemática realizada, sobre el desarrollo moral en contextos educativo, fueron seleccionados 167 artículos científicos, desarrollados desde el año 2010 hasta el año 2020, donde se abordó este aspecto y su relación con diversas variables o categorías de estudio detalladas a continuación. En efecto, esta revisión evidenció tendencias en la producción de conocimiento científico, intereses investigativos y avances en la comprensión del desarrollo moral a la luz del entorno educacional.

Los ejes temáticos identificados se categorizaron en tres segmentos de análisis, según el ciclo educativo investigado: 1) Los estudios asociados al desarrollo moral en la infancia, las prácticas pedagógicas asociadas a esta etapa vital, el rol del docente y del sistema familiar en la educación moral. 2) Los trabajos investigativos que evidenciaron resultados asociados al desarrollo moral en la adolescencia y educación secundaria, las practicas morales, interculturales e identitarias asociadas a esta etapa de desarrollo, el rol del docente, su incidencia en la educación moral y su relación con la realidad tecnológica. 3) Investigaciones relacionadas con el desarrollo moral, la educación superior y las prácticas profesionales, así como los procesos pedagógicos asociados con la educación moral en este nivel de formación.

\section{Desarrollo moral y educación inicial}

Respecto al desarrollo moral en la infancia, algunos estudios realizados en Europa han revelado una relación entre conductas positivas y razonamiento moral en escenarios de formación deportiva que involucraban ejercicios de cooperación y oposición, a su vez, el conocimiento moral se vio asociado al desarrollo de metáforas y escenarios educativos mediados por la cultura religiosa; seguidamente, se encontró una tendencia a las emociones morales asociadas a la culpa y la vergüenza en menores víctimas de bullying (HernándezMendo y Planchuelo, 2014; Romera et al., 2019; Taş, 2019). Por su parte, investigaciones efectuadas en América del Sur mostraron la influencia que ejercen los valores morales y las habilidades sociales en la convivencia y la democracia, así como en la percepción de los conflictos interpersonales en esta etapa vital (Barrios, 2016).

En cuanto a las prácticas pedagógicas morales, se han realizado investigaciones, en Oceanía, Europa y Asia, que evidencian la influencia que ejercen las creencias, las percepciones y los pensamientos del docente en la educación moral del menor, incluso, se han detectado preocupaciones o dilemas morales de los educadores asociados al desarrollo evolutivo, las necesidades, los sentimientos, la justicia y la honestidad, de los niños y niñas en sus aulas (Koc y Buzzelli, 2016; Lunn Brownlee et al., 2015; Temli et al., 2013; Walker et al., 2012). Luego, se detectó la relación positiva entre programas educativos con contenidos sociocientíficos y el incremento de la sensibilidad moral en la formación primaria, en paralelo con la autoría moral como elemento esencial en la formación de los docentes, es decir, 
la habilidad de observar, detectar, reflexionar y dialogar sobre cuestiones morales de su ejercicio profesional (Gertsen et al., 2017; E.-A. Kim et al., 2016).

En esta línea, se destaca el rol que ejerce la familia en el desarrollo moral en la infancia, a través de estudios desarrollados en Asia y América del Norte, donde se ha establecido que los estilos de crianza pueden predecir significativamente el desarrollo moral, así como su relación con el nivel socioeconómico y la educación materna (Ahmady et al., 2015); además, se evidenció la influencia del funcionamiento familiar en el desarrollo de emociones morales, como la empatía y la vergüenza, las cuales se asocian a una menor frecuencia de conductas agresivas en menores (Valdés Cuervo et al., 2016).

Otras investigaciones, realizadas en América del Norte y Europa, han analizado el juicio moral en la infancia, destacando la influencia de la cultura y evidenciando su influencia en la inclusión social de niños y niñas con necesidades educativas especiales, y menores en contextos de diversidad racial (Cameron et al., 2012; Gasser et al., 2013; Guerrero et al., 2017); descubriendo que, los infantes involucrados en aulas inclusivas mostraron más juicios y emociones morales que aquellos menores en aulas no inclusivas, expresando mayor simpatía y contacto con sus pares que presentaban características diversas (Gasser et al., 2013).

A su vez, estudios desarrollados en Europa, Asia y América, han indagado el desarrollo moral en la infancia y su transición a la adolescencia, encontrando que la identidad moral puede mediarse a través del deporte y la construcción de metas colectivas, lo que puede incidir en el rendimiento académico y en las aptitudes de interacción social (Jacobs et al., 2013; Proios, 2013). Otras investigaciones se enfocaron en la formación moral del docente, incidente en su liderazgo y pensamiento crítico, así como el efecto que ejercían las respuestas educativas ante las transgresiones morales, las regulaciones convivenciales y el clima escolar percibido, en el entendimiento y la motivación moral de los estudiantes (Doering et al., 2015; Lee, 2013; Martínez Moreno y Posada Gilède, 2014), incluso, se encontró que, los programas de convivencia escolar influyeron en el desarrollo y consolidación de valores morales y sociales (Avilés Martínez et al., 2019), también la vinculación a contextos situacionales interculturales incidieron en la evolución del razonamiento moral en esta etapa transicional (Lo et al., 2020).

\section{Desarrollo moral y educación secundaria}

Los estudios centrados en el desarrollo moral de adolescentes, realizados en Europa, Asia y América, han develado la relación negativa entre el uso prolongado de videojuegos violentos y la madurez socio moral, ante esto, se evidenció el papel que ejercen los contenidos de los videojuegos en el desarrollo moral (Bajovic, 2013; Hodge et al., 2019); en contraste, otro estudio evidenció la percepción de los adolescentes con respecto a su identidad moral, 
considerando esenciales la formación recibida en el sistema familiar y la interacción libre en los ambientes virtuales, aunque, revelaron la disonancia de estos aspectos con el contexto social (Fernández Cárdenas et al., 2015).

Seguidamente, se halló la influencia de la cultura en el desarrollo moral de este grupo etario (Christina y Jim, 2016), así como los mecanismos de desconexión moral y los comportamientos agresivos o considerados inadecuados (Hsu y Pan, 2018). Luego, se encontró que, guiar los comportamientos de los adolescentes a través de programas morales pudo promover el desarrollo de virtudes y la exteriorización adaptativa de conductas (Shek y Zhu, 2019), demostrando la importancia del sistema escolar en la formación moral y ética de los adolescentes, a través de aprendizajes transversales relevantes para la construcción de una sociedad democrática e incluyente (Lagos-Vargas y Keupuchur-Natalini, 2019).

En efecto, cabe mencionar los resultados investigativos enfocados en el desarrollo moral de los adolescentes en escenarios educativos, generados en Europa, donde se ha encontrado una relación causal entre el rendimiento académico y el nivel de desarrollo moral de estudiantes en educación secundaria (Díaz-Serrano, 2015a; Krause y Stark, 2016). A su vez, se demostró que la educación moral influyó en la comprensión de fenómenos asociados a la pobreza, el género y la educación, fortaleciendo las voces femeninas y el reconocimiento del rol materno como educadoras morales, así, estos programas morales pudieron fortalecer las relaciones respetuosas entre géneros (Arnot y Swartz, 2018). Otros estudios, realizados en América del Norte y Asia, mostraron la importancia de la participación de estudiantes en discursos transaccionales como forma de incrementar sus niveles de razonamiento moral (Nucci et al., 2015), evidenciaron los dilemas morales más frecuentes expresados por adolescentes, tales como, la autonomía, el respeto propio y mutuo, la confianza, la libertad y la tolerancia (Balakrishnan y Narvaez, 2016), la formación profesoral en pedagogía moral como medio para el fortalecimiento discursivo del educador e incremento en su conocimiento y dominio moral, influyentes en el razonamiento moral de sus estudiantes (Midgette et al., 2018), cabe entonces considerar los hallazgos de estudios previos sobre las diferencias significativas de género asociadas al nivel de razonamiento moral en docentes de secundaria y su tendencia a ubicarse en niveles de razonamiento convencional según la taxonomía de Kohlberg (Al-Shurai, 2012).

Otros aspectos por considerar se vinculan con el desarrollo del razonamiento moral, la toma de decisiones morales, el carácter y la identidad moral de los adolescentes, a través investigaciones realizadas en América del Norte, donde se constató el desarrollo del carácter moral de estudiantes a través de programas de asesoría y su relación con niveles significativamente altos de permanencia y vinculación comunitaria en su proceso académico (Seider et al., 2013), luego, se comprobó la influencia de servicios comunitarios en la construcción y consolidación de comportamientos cívicos e identidad moral (Jones et al., 2014). 
A su vez, con respecto a la cognición social de los adolescentes, el razonamiento moral ha ejercido una influencia en la toma de decisiones acertadas y se ha correlacionado con el desarrollo de funciones ejecutivas, tales como flexibilidad cognitiva, retroalimentación, razonamiento conceptual y fluidez verbal, evidenciado a través de variables como la edad, la inteligencia y la comunicación (Vera-Estay et al., 2015). Posteriormente, se observó la relación causal entre el comportamiento prosocial y la maduración del razonamiento moral, los valores e identidad moral (Shields et al., 2018). Por otro lado, estudios emergentes de Asia y Europa, presentan contribuciones a la comprensión de toma de decisiones morales y su incidencia en las emociones, destacando el rol de los pares en la atribución de estas emociones, así como el grado de razonamiento moral asociado a las normas sociales y la búsqueda del bien común (Hasegawa, 2016; Šramová, 2017).

Finalmente, se han destacado los estudios asociados al juicio y los comportamientos morales en la adolescencia, mediante estudios efectuados alrededor de Europa, América, África y Asia; dentro de estos se encuentran los hallazgos en estudiantes superdotados, quienes mostraban capacidades superiores en el juicio moral, en comparación con su grupo de edad, sin embargo esto no predecía su comportamiento moral (Tirri, 2011). Se evidenció desconfianza en los jóvenes ante la moralidad del adulto, expresando aspectos como el egoísmo, interés propio y enfoque en la esfera privada, cuestionando sus relaciones con la esfera pública (Imanishi et al., 2011). Por otro lado, hubo un incremento en el juicio moral de adolescentes y sus creencias epistemológicas, a través de un programa de educación ética basado en ciencia, tecnología y sociedad (Han y Jeong, 2014), así como los ajustes a lineamientos educativos basados en la teoría del desarrollo moral de Kohlberg (Moreira et al., 2020), la resiliencia e inteligencia espiritual de los estudiantes (Darvishzadeh y Bozorgi, 2016) y la acción moral de los educadores (Caetano y Dell'Agli, 2017). En contraste, se reveló la influencia de la heteromoralidad en la violencia homofóbica y cómo a través de esto se limitaba los derechos de gays y lesbianas, haciendo énfasis en el rol de la familia, la escuela y los procesos socioculturales en la prevención de la perpetración de estos hechos y los garantes de alianzas de apoyo a la justicia sexual (Bhana, 2013). Luego, se predijeron actitudes hacia la desigualdad social, los valores de justicia social y los juicios morales de los adolescentes, mediante la simpatía, la anticipación de las emociones morales y los factores contextuales en el desarrollo de estas actitudes (Malti et al., 2017).

\section{Desarrollo moral y educación superior}

Se ha abordado el desarrollo moral en la educación superior, alrededor de América, Europa y Asia, centrándose en ciencias de la salud, como medicina, enfermería, veterinaria, odontología, bioquímica y farmacia; ciencias administrativas, contables y económicas; ciencias educativas, como pedagogía y licenciaturas; disciplinas asociadas al deporte; ciencias aplicadas e ingenierías, ciencias sociales y jurídicas. 
Así, se observó una tendencia en el nivel convencional y el nivel post-convencional, sin hallar diferencias significativas entre el desarrollo moral y el grado de estudios alcanzados (Bordignon, 2011; Casas A et al., 2018; Chalmers et al., 2011; Nather, 2013; Torres et al., 2010; Zhao et al., 2018), en otros estudios se revelaron niveles altos o post-convencionales al iniciar estudios superiores (Bohm et al., 2014; Holland, 2011; Qaiser et al., 2012), y los niveles de sensibilidad moral oscilaron entre medio y alto (Tuvesson y Lützén, 2017). Aunque no se observaron diferencias significativas en sujetos que finalizaban su formación profesional, y no hubo evolución durante el ciclo académico (Holland, 2011; Murrell, 2014; Nacasato et al., 2016), incluso, se observó una tendencia regresiva durante este proceso, sugiriendo un incremento del fenómeno connotado como segmentación moral, más allá de la inhibición en el desarrollo moral (Chalmers et al., 2011; Hegazi y Wilson, 2013; Neves Feitosa et al., 2013; Prescott et al., 2014); así como niveles bajos de moralidad o mantenimiento de niveles previos, pese a recibir educación ética (Bronikowska y Korcz, 2019; de Souza et al., 2019; Esquerda et al., 2019; Forsha, 2017; Friedrich et al., 2017), revelando que, en tales casos, la educación universitaria no fomentó el desarrollo moral de los estudiantes (Hummel et al., 2018).

Lo anterior difiere de los hallazgos investigativos donde se expuso la evolución gradual del desarrollo moral asociado al desarrollo de competencias profesionales, involucrando estadios que van desde la transición y reconstrucción moral, hasta la interiorización moral (Gallagher, 2011; Hren et al., 2011; Ranjbar et al., 2018), a su vez, se reconoció la influencia de la formación en ética profesional y el desarrollo de competencias cognitivas socio-morales (Cheruvalath, 2019), así también se constataron mejoras significativas en el razonamiento moral de adultos con discapacidad intelectual, al participar de programas deportivos en escenarios educativos, para su adaptación e inclusión sociolaboral (Vílchez et al., 2018). Asimismo se observó que realizar estudios de posgrado influyó positivamente los niveles de competencia moral, sin embargo se identificó una disminución significativa asociada a prolongados ańos de práctica profesional (Agurto et al., 2017).

A su vez, el desarrollo moral de los universitarios se mostró influenciado por diversos factores e interacciones sociales (Monzon et al., 2010), relacionándose con la empatía afectiva, los valores personales (Juujärvi et al., 2012; Myyry et al., 2013; Skoe, 2010; Yuguero et al., 2019), la orientación comunitaria y libertaria (C. S. Lin et al., 2012), la comprensión de las necesidades de seguridad y las emociones del consultante y sus familias (Comrie, 2012; Peter et al., 2015), la motivación por el logro académico, el apoyo social percibido, la angustia psicológica (Al-Shuaibi et al., 2013), el desarrollo cognitivo, el auto concepto moral, la autoestima, los comportamientos prosociales y proambientales (Ploum et al., 2018; Rahnama et al., 2017; Vihos et al., 2019; Wang et al., 2013).

También influye como factores relevantes las experiencias anteriores co el clima escolar, las estrategias curriculares, las didácticas y los métodos de aprendizaje como debates, 
análisis de casos, dilemas morales, trabajos en equipo, actividades lúdicas y juegos de roles, el fortalecimiento del pensamiento crítico y reflexivo para el desarrollo profesional de la sensibilidad e identidad ética, la motivación moral (Baykara et al., 2015; Davis y Bergen, 2014; de Souza et al., 2019; Edwards et al., 2012; Esquerda et al., 2019; Kaplan et al., 2014; Lloyd-Hazlett y Foster, 2017; Maddineshat et al., 2019; V. S. M. Martins et al., 2020; Nacasato et al., 2016; Ploum et al., 2019; Ranjbar et al., 2017; Solum et al., 2016; Trobec y Starcic, 2015; Yuguero et al., 2019), el sistema familiar, el contexto y la condición socio cultural (Avila et al., 2018; Małgorzata Bronikowska et al., 2019; Davis y Bergen, 2014; Kurbanov et al., 2016; Phillips et al., 2020; Rzymska et al., 2014), las relaciones interpersonales, el acceso a la educación moral, la práctica e identidad profesional (Haghighat et al., 2020; Moreira et al., 2020; Sabatino et al., 2015; Van Stekelenburg et al., 2020), el rendimiento y los logros académicos (Corcoran y O'Flaherty, 2016; O'Flaherty y Gleeson, 2014), las características de la educación inicial, la integración de educación religiosa y moral (O'Flaherty y Gleeson, 2014), la actitud de los docentes y su interés por la formación en ciudadanía, desde la creatividad, la empatía, el diálogo, el respeto y la comprensión de los estudiantes a partir de sus valores espirituales, sentimientos morales e impulso en la toma de decisiones con juicios críticos en ambientes educativos motivadores (Enderle et al., 2018; Kurbanov et al., 2016; V. Martins et al., 2020; Sorensen et al., 2017).

Diversos estudios reportan la influencia de estrategias pedagógicas integradoras, en el incremento del razonamiento y la competencia moral (Bosco et al., 2010; Cummings et al., 2010), donde se vinculen discusiones de dilemas morales, análisis de casos (Khatiban et al., 2019; O'Flaherty y McGarr, 2014; Robles, 2013; Seiler et al., 2011; van Ommen et al., 2017), comprensión de narrativas y reconocimiento del currículo oculto (Hren et al., 2011; K. Y. Lin et al., 2014), escenarios interactivos de debate y argumentación, mediados por la tecnología o de forma presencial (Hewko et al., 2015; W.-J. Kim y Park, 2019; Özçinar, 2015), desde un enfoque de aprendizaje cooperativo centrado en el estudiante, para la promoción una cultura de disciplina académica (Syabuddin et al., 2020) y discusiones reflexivas sobre los conflictos éticos (Juujärvi et al., 2011).

La moralidad, las percepciones, dilemas y creencias de directivos y docentes ha sido objeto de estudio en este aspecto, destacando algunos factores como la sensibilidad, el juicio, el carácter y la motivación, vinculados al razonamiento moral de los líderes en educación (Greer et al., 2015), las competencias democráticas (Meza Pardo y Guerrero Chinga, 2016), el profesionalismo y la identidad ética (Amini et al., 2020; Hamilton y LaVoi, 2017), el reconocimiento de su rol en el proceso de desarrollo moral de sus estudiantes (de Alencar et al., 2014), las experiencias emocionales (Quinlan, 2019), las creencias, ideologías y actitudes, observándose una tendencia conservadora en culturas orientales (Asif et al., 2020).

Otras investigaciones se han centrado en el juicio moral, la habilidad para tomar decisiones, el comportamiento de citación, los conflictos y la angustia moral. Frente a lo 
cual, se encontraron altos niveles de juicio moral y puntuaciones post-convencionales en el género femenino y expertos en ética, en áreas de atención en salud (Swisher, 2010), incluso, se han planteado determinantes del comportamiento moral relacionados con el género, con respecto a los sistemas de poder, la toma de decisiones económicas, el liderazgo y probablemente con los comportamientos agresivos y criminales (Fumagalli et al., 2010), encontrandose además, diferencias de género en índices de razonamiento, motivación y aplicación moral (You y Bebeau, 2012).

Por su parte, se ha observado una influencia positiva entre las experiencias interculturales y el juicio moral (Narvaez y Hill, 2010), basadas en principios de empatía y apertura a la diversidad (Gerson y Neilson, 2014), crecimiento y práctica profesional, a través de espacios dialógicos donde se generen análisis de casos que involucren dilemas morales (Badro, 2011; Craig y Oja, 2013), cuestionamientos socráticos (Torabizadeh et al., 2018), comportamiento de citación y autocontrol (Hosseini Ahangari et al., 2020), formación con contenido ético, reflexivo y prosocial, que promueva el desarrollo de la sensibilidad, el juicio y el razonamiento moral (Park et al., 2012; Roche y Thoma, 2017; Verrinder y Phillips, 2015) requerido para la toma de decisiones éticas (Edwards et al., 2012) y morales (McCool y Bremser, 2014; Schmidt, 2014), lo que puede impulsar a cambios en los rasgos de personalidad (Bollich et al., 2016), de ahí la importancia de la formación moral en el incremento del razonamiento y el juicio moral (Cunha et al., 2017).

Se ha evidenciado una tendencia en las disciplinas de la salud a experimentar angustia moral, generando estados de agotamiento y erosión profesional (Wiggleton et al., 2010), revelando conflictos morales relacionados con compromisos laborales, factores humanos, deseo de éxito moral, resolución de problemas e inclinación al fracaso moral en términos de hacer algo que perciben como moralmente inadecuado (Camp y Sadler, 2019; Lazzari et al., 2020; Vartiainen, 2010), decisiones sobre el final de la vida, la muerte, la interacciones significativas entre el personal asistencial y los pacientes, la calidad de la atención, la privacidad, el trabajo en equipo y el acceso a la atención de la salud, es decir, factores personales, grupales e institucionales (Camp et al., 2018; Fernandes y Moreira, 2013), también se ha observado una inclinación hacia la justicia, la compasión y el cuidado de las personas (Bedregal et al., 2013; Quinn et al., 2012), tendiendo a la disonancia cognitiva entre sus percepciones de la atención ideal y la atención real que se le brinda a pacientes (Schrepel et al., 2019), además, no abordar la angustia moral desde la educación en salud, puede generar frustración profesional e ineficacia en la atención a pacientes (Aultman y Wurzel, 2014).

En contraste, la angustia moral en contextos formativos, ha generado estrategias que fomentan la deliberación moral, a nivel individual y colectiva, que involucran la defensa de principios y el diálogo, produciendo mecanismos compensatorios de preservación, objetividad y resistencia, influidos por los vínculos grupales, el liderazgo y las variabilidades 
individuales (Burton et al., 2020; de Barros et al., 2019); en consecuencia, el sistema educativo -a través de estrategias curriculares efectivas - requiere promover el desarrollo de las competencias morales, sociales y emocionales, religadas al conocimiento técnico científico (Pedraza-Chávez y Pérez-Ramírez, 2019), para ofrecer a sus educandos la oportunidad de fortalecer habilidades para tomar decisiones morales, autónomas, coherentes y eficientes (Tomaschewisk-Barlem, 2020).

\section{DISCUSIÓN}

A nivel global, la construcción episteme de conocimiento asociado al desarrollo moral se ha orientado predominantemente desde una postura empírico-analítica, la tendencia han sido investigaciones de tipo cuantitativo, ocupando el $63 \%$ de estudios rastreados y analizados, sobre un $32 \%$ de investigaciones cualitativas y un $5 \%$ de estudios mixtos. Con respecto al enfoque teórico, los estudios se han fundamentado en una postura cognitiva, su conceptualización se ha basado principalmente en los planteamientos de Piaget (1932; 1974; citado por Zerpa, 2007, p. 141), (Kohlberg, 1992).

En efecto, se ha observado una perspectiva moral asociada con la evolución progresiva de la cognición, mediante proceso madurativos y psicosociales, categorizando los hallazgos según los niveles de educación inicial, media y superior, ya que se evaluó el desarrollo intelectual desde la infancia hasta la adultez, buscando una explicación de los procesos cognitivos y estadios que pasa un individuo para conocer su realidad y alcanzar progresivamente su desarrollo moral (Rodríguez, 2016; Zerpa, 2007), a partir de principios universales que forjan su razonamiento y referencian sus juicios de valor (Rodríguez, 2016, p. 12).

La evidencia científica asociada al desarrollo moral y la educación inicial fue realizada aproximadamente en un 54\% en Europa; 23\% en América, del cual el 14\% se concentró en América del Norte y el $9 \%$ en América del Sur; otro 14\% se ubicó en Asia y el 9\% restante lo ocupó Oceanía. De estos hallazgos se analizó la relación entre la evolución de razonamiento moral, las conductas positivas y el deporte, la influencia de la cultura y las experiencias interculturales en la inclusión social y la consolidación de la identidad moral, los valores morales asociados a las relaciones interpersonales, gestionado en programas de convivencia escolar y educación moral, el rendimiento académico del educando, el abordaje institucional ante conductas transgresoras, la incidencia de las variabilidades del docente a nivel personal y profesional, donde se involucran dilemas morales y su rol en los programas formativos desde la sensibilidad moral (Barrios, 2016; Gasser et al., 2013; Hernández-Mendo y Planchuelo, 2014; Lo et al., 2020; Proios, 2013; Walker et al., 2012). Por otro lado, se reveló la influencia del sistema familia, su funcionalidad, su condición socioeconómica y educacional, las pautas de crianza y el manejo de las emociones morales en los infantes (Ahmady et al., 2015; Valdés Cuervo et al., 2016). 
Respecto al desarrollo moral en la educación media, el 52\% de los estudios se desarrolló en América, es decir, el 38\% se concentró en América del Norte y el 14\% en América del Sur; por su parte, un 24\% lo ocupó Asia, un 21\% se centró en Europa y un 3\% restante en África. Estas investigaciones revisadas mostraron la incidencia del contenido y el lapso de uso de los videojuegos en la madurez moral de los adolescentes, la relación entre la educación familiar, la cultura, las interacciones virtuales, las percepciones de los adolescentes y su identidad moral (Christina y Jim, 2016; Fernández Cárdenas et al., 2015; Hodge et al., 2019), la desconexión moral y su relación causal con los comportamientos agresivos, la influencia del rendimiento académico, la formación profesoral, los programas educativos morales y el sistema escolar en general, para el desarrollo del razonamiento moral y la construcción de ciudadanía (Díaz-Serrano, 2015a; Hsu y Pan, 2018; Lagos-Vargas y Keupuchur-Natalini, 2019; Midgette et al., 2018). Incluso, se han evidenciado relaciones entre el juicio moral, los comportamientos morales, las actitudes hacia la desigualdad y justicia social (Malti et al., 2017; Tirri, 2011); en esta línea, se constató la relación positiva entre la cognición social, los programas comunitarios, programas de asesoría académica, la toma de decisiones y el razonamiento moral, vinculados además a los comportamientos prosociales, regulación emocional, relaciones entre iguales y búsqueda del bien común (Jones et al., 2014; Shields et al., 2018; Šramová, 2017; Vera-Estay et al., 2015).

Las investigaciones realizadas sobre desarrollo moral en entornos de educación superior, se efectuaron en un 46\% en América, de los cuales el 31\% correspondió a América del Norte y el $15 \%$ a América del Sur; otro 34\% se ubicó en Europa, un 17\% en Asia y el 3\% en Oceanía. En estos estudios hubo un predominio de sujetos vinculados a las ciencias de la salud, educativas y administrativas, observando una tendencia en el desarrollo moral que osciló entre los estadios convencional y post-convencional (Torres et al., 2010; Zhao et al., 2018), observando discrepancias entre estudios que buscaban establecer la influencia de la formación universitaria en este aspecto, dado que, se ha hallado una tendencia regresiva en el proceso académico, explicado por el fenómeno de segmentación moral (Hegazi y Wilson, 2013); sin embargo otro estudio concluyó que, la educación moral y los escenarios deportivos con contenido ético profesional que podían influir favorablemente en el desarrollo moral de los estudiantes (Cheruvalath, 2019).

En efecto, se logró constatar en este grupo poblacional que su evolución moral está asociada a factores determinantes endógenos y exógenos, tales como: la empatía, el logro académico, el apoyo social percibido, la angustia moral, los comportamientos prosociales y el desarrollo cognitivo (Al-Shuaibi et al., 2013; Corcoran y O'Flaherty, 2016; Skoe, 2010; Vihos et al., 2019; Wang et al., 2013), las estrategias didácticas y curriculares, la familia, el entorno socio cultural, las características de los educadores y sus prácticas pedagógicas implementadas (Bosco et al., 2010; Małgorzata Bronikowska et al., 2019; Enderle et al., 2018; Kurbanov et al., 2016). 
En contraste, se centraron estudios en el juicio moral, la habilidad para tomar decisiones y la angustia moral (Swisher, 2010), relacionándolo con las experiencias interculturales, la apertura a la diversidad, el análisis de dilemas morales y la capacidad de la formación moral para enfrentar estos dilemas o conflictivas en la praxis profesional (Badro, 2011; Burton et al., 2020; Gerson y Neilson, 2014; Narvaez y Hill, 2010), destacando el papel fundamental que ejercen las instituciones educativas, la interacción docente - estudiante, en el establecimiento de sistemas de apoyo y crecimiento psicosocial, incidiendo incluso, en su rendimiento académico y logro disciplinar. De ahí la necesidad de cerrar la brecha entre el currículo tecnificado y el desarrollo humano de competencias morales, forjado desde el aprendizaje transformativo, desde la renovación a una pedagogía liberadora centrada en el estudiante, como ser indiviso capaz de construir realidades compartidas y ser ciudadano global (Al-Shuaibi et al., 2013; Avila et al., 2018; Kurbanov et al., 2016; Nacasato et al., 2016).

Finalmente, es necesario reconocer las limitaciones del estudio, como los idiomas excluidos en la revisión de los artículos, la selección de literatura únicamente de primera mano y electrónica, la exclusión de tesis o proyectos, y el énfasis en los escenarios educativos. Sin embargo este análisis sistemático ha revelado hallazgos y tendencias investigativas de alto impacto, generando un diálogo entre autores, al identificar puntos de encuentro y divergencias, segmentados por niveles de educación, lo que, como una contribución efectiva en la comprensión del desarrollo moral en escenarios formativos, interculturales y diversos. En efecto, este estudio ha cimentado los fundamentos para el abordaje de esta dimensión teórica en futuras investigaciones, que determinen el impacto curricular en el desarrollo moral, o el efecto de la educación moral en escolares y universitarios, lo que, establecerá un modelo o sistema efectivo de formación moral.

\section{CONCLUSIONES}

En síntesis, el abordaje del desarrollo moral en contextos educativos, además de ser un aspecto esencial en la formación de ciudadanos globales e interculturales, requiere un análisis integrado, que emerja de su complejidad y pluralidad de factores asociados, tales como las características individuales, familiares, sociales, históricas e institucionales, que convergen en el dinamismo de los espacios de aprendizaje y la triada docente-estudiantefamilia, como actores esenciales en el desarrollo de la competencia moral.

Así, las interacciones dinamizadas en los ecosistemas formativos, parten del reconocimiento de la influencia recíproca entre cada sujeto, constructor de realidades compartidas, lo que invita a la escuela de hoy, a la confrontación de los nuevos desafíos, a la adaptación del vertiginoso y continuo cambio que enfrenta, al desarrollo de un ser social capaz de convivir, de tomar decisiones, de generar relaciones humanas y contribuir 
satisfactoriamente en su comunidad, no solo desde lo disciplinar, académico o práctico, sino también desde sus capacidades indivisas, su propia conciencia y juicio ante la cotidianidad, es decir, un ser humano no escindido entre lo intelectual y moral. De ahí la relevancia de esta revisión sistemática, a nivel social y educativo.

Esta revisión es un punto de partida, las preguntas problematizadoras que surjan de los hallazgos descritos contribuirán a la construcción de conocimiento científico de alto impacto, pertinente, relevante y adaptado al contexto formativo en que se desarrolla y al cual se debe. Emerge entonces un incipiente desafío en la generación de conocimiento adaptado a las realidades que se vivencian, integrado al hacer, al ser y al saber convivir con la otredad. De este modo, los actores inmersos en los procesos educativos están llamados a promover dinámicas que posibiliten formar ciudadanía, más allá de la tecnificación de saberes, se requiere apostar por un ser creativo, crítico, reflexivo, capaz de tomar decisiones a partir de sus lógicas colectivas e indivisas, capaz de convivir entre antagónicos e intereses compartidos, libre y responsable a la vez.

\section{REFERENCIAS}

Agurto, M., Telloa, D., Elgueta, A., Larrea, R., Minaeff, T., Miranda, A., Parodi, E., Salas, J. M., Vukusich, A., Llanos, S., Daza, P., y López, S. (2017). Assessment of moral competence of physicians . Revista Médica de Chile, 145(9), 1122-1128. https://doi. org/10.4067/s0034-98872017000901122

Ahmady, A. K., Nia, K. R., Sahraei, F., y Sadri, F. (2015). Study of relationship between parenting styles and moral development. Advances in Environmental Biology, 9(3), 738-745.

Al-Shuaibi, J., Hamdan-Mansour, A. M., y Haourani, E. M. (2013). Correlates of moral development among university students in Jordan. Life Science Journal, 10(4), 26072612.

Al-Shurai, S. (2012). An Empirical Investigation of the Moral Judgment Development of a Sample of High School Kuwaiti Teachers. Education, 133(2), 340-348.

Amini, D., Najafi, S., y Behin, B. (2020). Exploring EFL teachers' moral identity: the case of iranian teachers. Journal of Teacher Education for Sustainability, 22(1), 111-123. https://doi.org/10.2478/jtes-2020-0009

Arnot, M., y Swartz, S. (2018). Gender respect: Empirical insights for (moral) educators about women's struggles for respect in the Global South. Journal of Moral Education, 47(4), 481-497. https://doi.org/10.1080/03057240.2018.1433644

Asif, T., Guangming, O., Haider, M. A., Colomer, J., Kayani, S., y ul Amin, N. (2020). Moral education for sustainable development: Comparison of university teachers' 
perceptions in China and Pakistan. Sustainability (Switzerland), 12(7), 3014. https://doi.org/10.3390/su12073014

Aultman, J., y Wurzel, R. (2014). Recognizing and alleviating moral distress among obstetrics and gynecology residents. Journal of Graduate Medical Education, 6(3), 457-462. https://doi.org/10.4300/JGME-D-13-00256.1

Avila, L. I., da Silveira, R. S., de Figueiredo, P. P., Mancia, J. R., Gonçalves, N. G. da C., y Barlem, J. G. T. (2018). Moral construction of undergraduate nursing students to promote care humanization. Texto e Contexto Enfermagem, 27(3), 1-9. https://doi. org/10.1590/0104-070720180004790015

Avilés Martínez, J. M., De Stefano Menin, M. S., y Rossi Tavares, M. (2019). Evaluation of sociomoral values in students. Comparative research between Brazil and Spain. Revista de Educacion, 2019(384), 191-219. https://doi.org/10.4438/1988-592XRE-2019-384-410

Badro, V. (2011). Dilemmas of conscience in the practice of medicine: a phenomenological study. Narrative Inquiry in Bioethics, 1(3), 171-188. https://doi.org/10.1353/ nib.2011.0054

Bajovic, M. (2013). Violent video gaming and moral reasoning in adolescents: Is there an association? Educational Media International, 50(3), 177-191. https://doi.org/10.10 80/09523987.2013.836367

Baker, J. D. (2016). The purpose, process, and methods of writing a literature review. AORN journal, 103(3), 265-269. https://doi.org/10.1016/j.aorn.2016.01.016

Balakrishnan, V., y Narvaez, D. (2016). A reconceptualisation of vygotsky's ZPD into ZCD in teaching moral education in secondary schools using real-life dilemmas. Cogent Education, 3(1). 1-15. https://doi.org/10.1080/2331186X.2016.1142925

Barrios, A. (2016). Concepciones de conflictos interpersonales y desarrollo moral en la educación infantil brasileña. Revista de Psicología, 34(2), 261-291. https://doi. org/10.18800/psico.201602.002

Baykara, Z. G., Demir, S. G., y Yaman, S. (2015). The effect of ethics training on students recognizing ethical violations and developing moral sensitivity. Nursing Ethics, 22(6), 661-675. https://doi.org/10.1177/0969733014542673

Bedregal, P., León, T., Shand, B., y Mosso, L. (2013). Survey on moral judgment criteria among medical students . Revista Médica de Chile, 141(6), 704-709. https://doi. org/10.4067/S0034-98872013000600003

Bhana, D. (2013). Parental views of morality and sexuality and the implications for South African moral education. Journal of Moral Education, 42(1), 114-128. https://doi.or g/10.1080/03057240.2012.737314 
Bohm, K. C., Van Heest, T., Gioe, T. J., Agel, J., Johnson, T. C., y Van Heest, A. (2014). Assessment of moral reasoning skills in the orthopaedic surgery resident applicant. The Journal of Bone and Joint Surgery, 96(17), e151. https://doi.org/10.2106/ JBJS.M.00706

Bollich, K. L., Hill, P. L., Harms, P. D., y Jackson, J. J. (2016). When friends' and society's expectations collide: A longitudinal study of moral decision-making and personality across college. PloS One, 11(1), e0146716. https://doi.org/10.1371/journal. pone. 0146716

Bordignon, N. A. (2011). Consequences of moral Kohlberg's moral development levels in higher education. Revista Lasallista de Investigacion, 8(1), 16-27. http://www.scielo. org.co/pdf/rlsi/v8n1/v8n1a03.pdf

Bosco, S. M., Melchar, D. E., Beauvais, L. L., y Desplaces, D. E. (2010). Teaching business ethics: The effectiveness of common pedagogical practices in developing students' moral judgment competence. Ethics and Education, 5(3), 263-280. https://doi.org/ 10.1080/17449642.2010.533049

Bronikowska, M, y Korcz, A. (2019). The level of moral competences of pre-service PE teachers - A reason to worry? Biomedical Human Kinetics, 11(1), 19-27. https://doi. org/10.2478/bhk-2019-0003

Bronikowska, Małgorzata, Korcz, A., Krzysztoszek, J., y Bronikowski, M. (2019). How years of sport training influence the level of moral competences of physical education and sport students. BioMed Research International, 2019, 1-10. https:// doi.org/10.1155/2019/4313451

Burton, M., Caswell, H., Porter, C., Mott, S., y DeGrazia, M. (2020). Moral Distress: Defined and described by neonatal and pediatric critical care nurses in a quaternary care free-standing pediatric hospital. Dimensions of Critical Care Nursing, 39(2), 101-109. https://doi.org/10.1097/DCC.0000000000000403

Caetano, L. M., y Dell'Agli, B. A. V. (2017). Teacher's moral educational conceptions. Arquivos Brasileiros de Psicología, 69(3), 167-182. http://pepsic.bvsalud.org/pdf/ arbp/v69n3/12.pdf

Cameron, C. A., Lau, C., Fu, G., y Lee, K. (2012). Development of children's moral evaluations of modesty and self-promotion in diverse cultural settings. Journal of Moral Education, 41(1), 61-78. https://doi.org/10.1080/03057240.2011.617414

Camp, M. E., Jeon-Slaughter, H., Johnson, A. E., y Sadler, J. Z. (2018). Medical student reflections on geriatrics: Moral distress, empathy, ethics and end of life. Gerontology and Geriatrics Education, 39(2), 235-248. https://doi.org/10.1080/02701960.2017 .1391804 
Camp, M., y Sadler, J. (2019). Moral distress in medical student reflective writing. AJOB Empirical Bioethics, 10(1), 70-78. https://doi.org/10.1080/23294515.2019.15703 85

Casas A, L. F., Ibáńez A, L. E., y Parra, D. I. (2018). Desarrollo moral de los estudiantes de un programa de enfermería. Salud UIS, 50(3), 247-256. https://doi.org/10.18273/ revsal.v50n3-2018009

Chalmers, P., Dunngalvin, A., y Shorten, G. (2011). Reflective ability and moral reasoning in final year medical students: a semi-qualitative cohort study. Medical Teacher, 33(5), e281-9. https://doi.org/10.3109/0142159X.2011.558531

Cheruvalath, R. (2019). Does studying "ethics» improve engineering students' metamoral cognitive skills? Science and Engineering Ethics, 25(2), 583-596. https://doi. org/10.1007/s1 1948-017-0009-x

Christina, L.-H. C., y Jim, Q. C. (2016). A scenario-based exploratory study of cognitive moral development in information ethics. International Journal of Information Systems and Change Management, 8(1), 60-78. https://doi.org/10.1504/ IJISCM.2016.077953

Comrie, R. W. (2012). An analysis of undergraduate and graduate student nurses' moral sensitivity. Nursing Ethics, 19(1), 116-127. https://doi. org/10.1177/0969733011411399

Corcoran, R. P., y O'Flaherty, J. (2016). Examining the impact of prior academic achievement on moral reasoning development among college students: A growth curve analysis. Journal of Moral Education, 45(4), 433-448. https://doi.org/10.1080 /03057240.2016.1230051

Craig, P. J., y Oja, S. N. (2013). Moral judgement changes among undergraduates in a capstone internship experience. Journal of Moral Education, 42(1), 43-70. https:// doi.org/10.1080/03057240.2012.677603

Cummings, R., Maddux, C. D., Cladianos, A., y Richmond, A. (2010). Moral reasoning of education students: The effects of direct instruction in moral development theory and participation in moral dilemma discussion. Teachers College Record, 112(3), 621-644. https://www.learntechlib.org/p/108750/

Cunha, M., Durte, J., Silva, E., Silva, D., y Pina, J. (2017). Reasoning and moral judgement in higher education students: Reality and challenge. Turkish Online Journal of Educational Technology, (2017). 812-824.

Darvishzadeh, K., y Bozorgi, Z. D. (2016). The relationship between resilience, psychological hardiness, spiritual intelligence, and development of the moral judgement of the female students. Asian Social Science, 12(3), 170-176. https://doi.org/10.5539/ass. v12n 3 p 170 
Davis, D. R., y Bergen, D. (2014). Relationships among play behaviors reported by college students and their responses to moral issues: A pilot study. Journal of Research in Childhood Education, 28(4), 484-498. https://doi.org/10.1080/02568543.2014.94 4721

de Alencar, H. M., De Marchi, B. F., Moraes Couto, L. L., Romaneli, M. S., y de Lima, M. G. (2014). Educação em valores morais: Juízos de profissionais no contexto escolar. Psicologia Escolar e Educacional, 18(2), 255-264. https://doi.org/10.1590/21753539/2014/0182742

de Barros, A. M., Ramos, F. R. S., Barth, P. O., Brito, M. J. M., Rennó, H. M. S., y Rocha, J. M. (2019). The moral deliberation process of college nursing professors in view of moral distress. Nurse Education Today, 73, 71-76. https://doi.org/10.1016/j. nedt.2018.11.014

de Souza, E. S., Serafim, M. C., y Santos, L. S. (2019). A contribuição do ensino de ética no desenvolvimento da competência moral de discentes em administração pública. education policy analysis archives, 127(104), 1-30. https://doi.org/10.14507/ epaa.27.4088

Díaz-Serrano, J. (2015b). El desarrollo del juicio moral en Kohlberg como factor condicionante del rendimiento académico en ciencias sociales de un grupo de estudiantes de educación secundaria. Revista Electrónica Educare, 19(3), 72-85. https://doi.org/10.15359/ree.19-3.8

Doering, B., Bergmann, M. C., y Hanslmaier, M. (2015). The importance of school for the development of moral motivation: Are the effects gender-specific? . Psychologie in Erziehung und Unterricht, 62(2), 136-146. https://doi.org/10.2378/peu2015.art07d

Durkheim, É. (1895). Educación y sociología. Ediciones Península.

Edwards, I., Kessel, G. van, Jones, M., Beckstead, J., y Swisher, L. L. (2012). The development of moral judgment and organization of ethical knowledge in final year physical therapy students. Physical Therapy Reviews, 17(3), 157-166. https://doi. org/10.1179/1743288X12Y.0000000001

Enderle, C. F., Silveira, R. S. D., Dalmolin, G. L., Lunardi, V. L., Avila, L. I., y Dominguez, C. C. (2018). Teaching strategies: promoting the development of moral competence in undergraduate students. Revista brasileira de enfermagem, 71, 1650-1656. https:// doi.org/10.1590/0034-7167-2017-0704

Esquerda, M., Pifarré, J., Roig, H., Busquets, E., Yuguero, O., y Viñas, J. (2019). Assessing bioethics education: Teaching to be virtuous doctors or just doctors with practical ethical skills. Atencion primaria, 51(2), 99-104. https://doi.org/10.1016/j. aprim.2017.05.018 
Fernandes, M. I. D., y Moreira, I. M. P. B. (2013). Ethical issues experienced by intensive care unit nurses in everyday practice. Nursing Ethics, 20(1), 72-82. https://doi. org/10.1177/0969733012452683

Fernández Cárdenas, J., Yáñez Figueroa, J., y Muñoz Villarreal, E. (2015). Prácticas morales y normas de netiqueta en las interacciones virtuales de los estudiantes de educación secundaria. Innovación Educativa, 15(69), 57-71. http://www.scielo.org.mx/pdf/ie/ v15n69/v15n69a5.pdf

Forsha, S. (2017). Virtue and moral development, changing ethics instruction in business school education. College Student Journal, 51(3), 429-443.

Friedrich, O., Hemmerling, K., Kuehlmeyer, K., Nörtemann, S., Fischer, M., y Marckmann, G. (2017). Principle-based structured case discussions: Do they foster moral competence in medical students? - A pilot study. BMC Medical Ethics, 18(21), 1-8. https://doi.org/10.1186/s12910-017-0181-1

Fumagalli, M., Ferrucci, R., Mameli, F., Marceglia, S., Mrakic-Sposta, S., Zago, S., Lucchiari, C., Consonni, D., Nordio, F., Pravettoni, G., Cappa, S., y Priori, A. (2010). Gender-related differences in moral judgments. Cognitive Processing, 11(3), 219-226. https://doi.org/10.1007/s10339-009-0335-2

Gallagher, C. T. (2011). Assessment of levels of moral reasoning in pharmacy students at different stages of the undergraduate curriculum. International Journal of Pharmacy Practice, 19(5), 374-380. https://doi.org/10.1111/j.2042-7174.2011.00121.x

Gasser, L., Malti, T., y Buholzer, A. (2013). Children's moral judgments and moral emotions following exclusion of children with disabilities: Relations with inclusive education, age, and contact intensity. Research in Developmental Disabilities, 34(3), 948-958. https://doi.org/10.1016/j.ridd.2012.11.017

Gerson, M. W., y Neilson, L. (2014). The importance of identity development, principled moral reasoning, and empathy as predictors of openness to diversity in emerging adults. SAGE Open, 4(4). https://doi.org/10.1177/2158244014553584

Gertsen, R., Schaap, H., y Bakker, C. (2017). Moral authorship of novice teachers in primary education. Teachers and Teaching: Theory and Practice, 23(5), 570-582. https://doi.org/10.1080/13540602.2016.1218842

Greer, J. L., Searby, L. J., y Thoma, S. J. (2015). Arrested development? Comparing educational leadership students with national norms on moral reasoning. Educational Administration Quarterly, 51(4), 511-542. https://doi. org/10.1177/0013161X14539807

Guerrero, S., Elenbaas, L., Enesco, I., y Killen, M. (2017). La confianza de los niños en el consenso depende del contexto: Convencional vs. moral. Anales 
de Psicología, 33(1), 142-151. https://scielo.isciii.es/scielo.php?script=sci_ arttext\&pid=S0212-97282017000100017

Haghighat, S., Borhani, F., y Ranjbar, H. (2020). Is there a relationship between moral competencies and the formation of professional identity among nursing students? BMC Nursing, 19(1). https://doi.org/10.1186/s12912-020-00440-y

Hamilton, M. G. B., y LaVoi, N. M. (2017). Ethical professional identity and the development of moral exemplar collegiate coaches. Journal of Moral Education, 46(2), 114-128. https://doi.org/10.1080/03057240.2017.1313724

Han, H., y Jeong, C. (2014). Improving epistemological beliefs and moral judgment through an STS-based science ethics education program. Science and Engineering Ethics, 20(1), 197-220. https://doi.org/10.1007/s11948-013-9429-4

Hasegawa, M. (2016). Development of moral emotions and decision-making from childhood to young adulthood. Journal of Moral Education, 45(4), 387-399. https:// doi.org/10.1080/03057240.2016.1213708

Hegazi, I., y Wilson, I. (2013). Medical education and moral segmentation in medical students. Medical Education 47(10), 1022-1028. https://doi.org/10.1111/ medu. 12252

Hernández-Mendo, A., y Planchuelo, L. (2014). El incremento del desarrollo moral en las clases de educación física. Revista Iberoamericana de Psicología del Ejercicio y el Deporte, 9(2), 369-392.

Hewko, S. J., Cooper, S. L., y Cummings, G. G. (2015). Strengthening moral reasoning through dedicated ethics training in dietetic preparatory programs. Journal of Nutrition Education and Behavior, 47(2), 156-161. https://doi.org/10.1016/j. jneb.2014.10.001

Hodge, S. E., Taylor, J., y McAlaney, J. (2019). It's doubled edged: The positive and negative relationships between the development of moral reasoning and video game play among adolescents. Frontiers in Psychology, 10. https://doi.org/10.3389/ fpsyg.2019.00028

Holland, L. (2011). Do higher education computing degree courses develop the level of moral judgement required from a profession? Journal of Information, Communication and Ethics in Society, 9(2), 116-126. https://doi.org/10.1108/14779961111148631

Hosseini Ahangari, Z., Hosseini Ahangari, A., y Alaae Arani, M. (2020). Teaching how to cite scientific articles: a study of citation deviation in citing «multi-authored papers» by top authors from Iranian Universities of Medical Sciences in 2017. Gastroenterology and Hepatology from Bed to Bench, 13(3), 258-263. 
Hren, D., Marušić, M., y Marušić, A. (2011). Regression of moral reasoning during medical education: Combined design study to evaluate the effect of clinical study years. PLoS ONE, 6(3). https://doi.org/10.1371/journal.pone.0017406

Hsu, W.-T., y Pan, Y.-H. (2018). Moral disengagement and student misbehavior in physical education. Journal of Sports Science and Medicine, 17(3), 437-444.

Hummel, K., Pfaff, D., y Rost, K. (2018). Does Economics and Business Education Wash Away Moral Judgment Competence? Journal of Business Ethics, 150(2), 559-577. https://doi.org/10.1007/s10551-016-3142-6

Imanishi, H. A., Passarelli, V. L. dos S., y de la Taille, Y. J. J. M. R. (2011). Morals in the adult world: How youth see their contemporary elders. Educacao e Pesquisa, 37(4), 743-760. https://doi.org/10.1590/s1517-97022011000400005

Jacobs, F., Knoppers, A., y Webb, L. (2013). Making sense of teaching social and moral skills in physical education. Physical Education and Sport Pedagogy, 18(1), 1-14. https://doi.org/10.1080/17408989.2011.621118

Jones, J. N., Warnaar, B. L., Bench, J. H., y Stroup, J. (2014). Promoting the development of moral identity, behavior, and commitment in a social action program. Journal of Peace Education, 11(2), 225-245. https://doi.org/10.1080/17400201.2014.898626

Juujärvi, S., Myyry, L., y Pesso, K. (2012). Empathy and values as predictors of care development. Scandinavian Journal of Psychology, 53(5), 413-420. https://doi. org/10.1111/j.1467-9450.2012.00961.x

Juujärvi, S., Pesso, K., y Myyry, L. (2011). Care-based ethical reasoning among first-year nursing and social services students. Journal of Advanced Nursing, 67(2), 418-427. https://doi.org/10.1111/j.1365-2648.2010.05461.x

Kaplan, U., Crockett, C. E., y Tivnan, T. (2014). Moral motivation of college students through multiple developmental structures: Evidence of intrapersonal variability in a complex dynamic system. Motivation and Emotion, 38(3), 336-352. https://doi. org/10.1007/s11031-013-9391-0

Khatiban, M., Falahan, S. N., Amini, R., Farahanchi, A., y Soltanian, A. (2019). Lecturebased versus problem-based learning in ethics education among nursing students. Nursing Ethics, 26(6), 1753-1764. https://doi.org/10.1177/0969733018767246

Kim, E.-A., Park, E.-Y., Lim, S.-M., y Yang, I.-H. (2016). Development and application of an education program based on socio-scientific issues for enhancing students' understanding of the nature of science and moral sensitivity. Information, 19(8B), 3427-3432. 
Kim, W.-J., y Park, J.-H. (2019). The effects of debate-based ethics education on the moral sensitivity and judgment of nursing students: A quasi-experimental study. Nurse Education Today, 83, 104200. https://doi.org/10.1016/j.nedt.2019.08.018

Koc, K., y Buzzelli, C. A. (2016). Turkish teachers' accounts of moral dilemmas in early childhood settings. Journal of Early Childhood Research, 14(1), 28-42. https://doi. org/10.1177/1476718X14523751

Kohlberg, L. (1992). Psicología del desarrollo moral. Desclée de.

Kohlberg, L., y Mayer, R. (1972). Development as the Aim of Education. Harvard Educational Review, 42(4), 449-496. https://doi.org/10.17763/haer.42.4.kj6q8743r3j00j60

Krause, U.-M., y Stark, R. (2016). Promoting sociomoral learning: Comparison of two teaching methods . Psychologie in Erziehung und Unterricht, 63(2), 81-90. https:// doi.org/10.2378/peu2016.art10d

Kurbanov, R. A., Nikonova, E. I., Gurbanov, R. A., Svechnikova, N. V, Tumarov, K. B., y Marin, E. M. (2016). Anthropological methods of formation of university students' spiritual and moral culture. International Journal of Environmental and Science Education, 11(18), 11807-11817.

Lagos-Vargas, R., y Keupuchur-Natalini, C. (2019). Development of moral competence in secondary school students: A reflection about the ethical training in school. Opcion, 35(90), 1301-1321.

Lazzari, T., Terzoni, S., Destrebecq, A., Meani, L., Bonetti, L., y Ferrara, P. (2020). Moral distress in correctional nurses: A national survey. Nursing Ethics, 27(1), 40-52. https://doi.org/10.1177/0969733019834976

Lee, C.-M. A. (2013). Qualitative inquiry on principals' moral development and their building of morality and character in schools. Journal of Research in Education Sciences, 58(1), 117-147. https://doi.org/10.3966/2073753X2013035801005

Liberati, A., Altman, D. G., Tetzlaff, J., Mulrow, C., Gotzsche, P. C., Ioannidis, J. P. A., y Moher, D. (2009). The PRISMA statement for reporting systematic reviews and meta-analyses of studies that evaluate healthcare interventions: Explanation and elaboration. British Medical Journal, 339, b2700. https://doi.org/10.1136/bmj. b2700

Lin, C. S., Tsou, K. I., Cho, S. L., Hsieh, M. S., Wu, H. C., y Lin, C. H. (2012). Is medical students' moral orientation changeable after preclinical medical education? Journal of Medical Ethics, 38(3), 168-173. https://doi.org/10.1136/medethics-2011-100092

Lin, K. Y., Anspach, R. R., Crawford, B., Parnami, S., Fuhrel-Forbis, A., y De Vries, R. G. (2014). What must I do to succeed?: narratives from the US premedical 
experience. Social Science y Medicine, 119, 98-105. https://doi.org/10.1016/j. socscimed.2014.08.017

Lloyd-Hazlett, J., y Foster, V. A. (2017). Student counselors' moral, intellectual, and professional ethical identity development. Counseling and Values, 62(1), 90-105. https://doi.org/10.1002/cvj.12051

Lo, J. H.-Y., Fu, G., Lee, K., y Cameron, C. A. (2020). Development of moral reasoning in situational and cultural contexts. Journal of Moral Education, 49(2), 177-193. https://doi.org/10.1080/03057240.2018.1563881

Londoño, O., Maldonado, L., y Calderón, L. (2014). Guía para construir estados del arte. International Corporation Of Networks Of Knowledge.

Lunn Brownlee, J., Johansson, E., Cobb-Moore, C., Boulton-Lewis, G., Walker, S., y Ailwood, J. (2015). Epistemic beliefs and beliefs about teaching practices for moral learning in the early years of school: relationships and complexities. Education 3-13, 43(2), 164-183. https://doi.org/10.1080/03004279.2013.790458

Maddineshat, M., Yousefzadeh, M. R., Mohseni, M., Maghsoudi, Z., y Ghaffari, M. E. (2019). Teaching ethics using games: Impact on Iranian nursing students' moral sensitivity. Indian Journal of Medical Ethics, 4(1), 14-20. https://doi.org/10.20529/ IJME.2018.056

Malti, T., Dys, S. P., Cui, L., y Buchmann, M. (2017). Moral and social antecedents of young adults' attitudes toward social inequality and social justice values. Longitudinal and Life Course Studies, 8(1), 5-19. https://doi.org/10.14301/llcs.v8i1.397

Martínez Moreno, C. A., y Posada Gilède, R. (2014). Evaluación de Respuestas de Dominio Apropiado e Inapropiado a Transgresiones Socio-Morales en el Contexto Educativo. Revista Colombiana de Psicología, 23(1), 119-133. https://doi.org/10.15446/rcp. v23n 1.40030

Martins, V. S. M., Santos, C. M. N. C., Bataglia, P. U. R., y Duarte, I. M. R. F. (2020). The teaching of ethics and the moral competence of medical and nursing students. Health Care Analysis. https://doi.org/10.1007/s10728-020-00401-1

Martins, V., Santos, C., y Duarte, I. (2020). Bioethics education and the development of nursing students' moral competence. Nurse Education Today, 95, 104601. https:// doi.org/10.1016/j.nedt.2020.104601

McCool, L. M., y Bremser, J. A. (2014). Individual differences in disgust sensitivity do not influence moral reasoning, but a discipline-specific ethics course does. SAGE Open, 4(3). https://doi.org/10.1177/2158244014541774 
Meza Pardo, M., y Guerrero Chinga, A. (2016). Competencia moral-democrática en futuros profesores de Pedagogía en educación básica en Chile ¿Importa la formación inicial docente? Perfiles Educativos, 38(154), 41-56.

Midgette, A. J., Ilten-Gee, R., Powers, D. W., Murata, A., y Nucci, L. (2018). Using lesson study in teacher professional development for domain-based moral education. Journal of Moral Education, 47(4), 498-518. https://doi.org/10.1080/03057240.2 018.1445982

Moher D, Liberati A, Tetzlaff J, Altman DG, The PRISMA Group (2009). Preferred Reporting Items for Systematic Reviews and Meta-Analyses: The PRISMA Statement. PLoS Med 6(7): e1000097. https://doi.org/10.1371/journal.pmed.1000097

Monzon, J. E., Ariasgago, O. L., y Monzon-Wyngaard, A. (2010). Assessment of moral judgment of BME and other health sciences students. Annual International Conference of the IEEE Engineering in Medicine and Biology. 2963-2966. https://doi. org/10.1109/IEMBS.2010.5626266

Moreira, D. de A., Ferraz, C. M. L. C., Costa, I. P. da, Amaral, J. M., Lima, T. T., y Brito, M. J. M. (2020). Professional practice of nurses and influences on moral sensitivity. Revista gaucha de enfermagem, 41, e20190080. https://doi.org/10.1590/19831447.2019.20190080

Murrell, V. S. (2014). The failure of medical education to develop moral reasoning in medical students. International journal of medical education, 5, 219-225. https://doi. org/10.5116/ijme.547c.e2d1

Myyry, L., Juujärvi, S., y Pesso, K. (2013). Change in values and moral reasoning during higher education. European Journal of Developmental Psychology, 10(2), 269-284. https://doi.org/10.1080/17405629.2012.757217

Nacasato, R. P., Bomfim, R. A., y De-Carli, A. D. (2016). Ethical and moral development: Aspects relating to professional training in dentistry . $R G O$ Revista Gaucha de Odontologia, 64(1), 43-49. https://doi.org/10.1590/1981863720160001000063056

Narvaez, D., y Hill, P. L. (2010). The relation of multicultural experiences to moral judgment and mindsets. Journal of Diversity in Higher Education 3(1), 43-55. https://doi.org/10.1037/a0018780

Nather, F. (2013). Exploring the impact of formal education on the moral reasoning abilities. College Student Journal, 47(3), 470-478.

Neves Feitosa, H., Rego, S., Unger Raphael Bataglia, P., Castelo Branco Sancho, K. F., Rego, G., y Nunes, R. (2013). Moral judgment competence of medical students: A transcultural study. Advances in Health Sciences Education, 18. 1067-1085. https:// doi.org/10.1007/s10459-013-9449-5 
Nucci, L., Creane, M. W., y Powers, D. W. (2015). Integrating moral and social development within middle school social studies: a social cognitive domain approach. Journal of Moral Education, 44(4), 479-496. https://doi.org/10.1080/03057240.2015.10873 91

O'Flaherty, J., y Gleeson, J. (2014). Longitudinal study of levels of moral reasoning of undergraduate students in an Irish university: the influence of contextual factors. Irish Educational Studies, 33(1), 57-74. https://doi.org/10.1080/03323315.2013.8 74544

O'Flaherty, J., y McGarr, O. (2014). The use of case-based learning in the development of student teachers' levels of moral reasoning. European Journal of Teacher Education, 37(3), 312-330. https://doi.org/10.1080/02619768.2013.870992

Okoli, C., y Schabram, K. (2010). A guide to conducting a systematic literature review of information systems research. SSRN Electronic Journal, 10(26). https://doi. org/10.2139/ssrn.1954824

Özçinar, H. (2015). Scaffolding computer-mediated discussion to enhance moral reasoning and argumentation quality in pre-service teachers. Journal of Moral Education, 44(2), 232-251. https://doi.org/10.1080/03057240.2015.1043875

Park, M., Kjervik, D., Crandell, J., y Oermann, M. H. (2012). The relationship of ethics education to moral sensitivity and moral reasoning skills of nursing students. Nursing Ethics, 19(4), 568-580. https://doi.org/10.1177/0969733011433922

Pedraza-Chávez, J., y Pérez-Ramírez, J. (2019). Level of moral judgment in students of the medical career of a public university . Medicina Interna de México, 35(6), 885-895. https://doi.org/10.24245/mim.v35i6.3416

Peter, E., Mohammed, S., y Simmonds, A. (2015). Sustaining hope as a moral competency in the context of aggressive care. Nursing Ethics, 22(7), 743-753. https://doi. org/10.1177/0969733014549884

Phillips, C. J. C., Çöl, R., I Zmirli, S., y Verrinder, J. M. (2020). Comparison of the moral sensitivity, judgment, and actions of australian and turkish veterinary students in relation to animal ethics issues. Journal of Veterinary Medical Education, 47(1), 8-17. https://doi.org/10.3138/jvme.1117-178r1

Ploum, L., Blok, V., Lans, T., y Omta, O. (2018). Exploring the relation between individual moral antecedents and entrepreneurial opportunity recognition for sustainable development. Journal of Cleaner Production, 172, 1582-1591. https:// doi.org/10.1016/j.jclepro.2017.10.296

Ploum, L., Blok, V., Lans, T., y Omta, O. (2019). Educating for self-interest or -transcendence? An empirical approach to investigating the role of moral competencies in opportunity 
recognition for sustainable development. Business Ethics, 28(2), 243-260. https:// doi.org/10.1111/beer.12214

Prescott, J., Becket, G., y Wilson, S. E. (2014). Moral development of first-year pharmacy students in the United Kingdom. American Journal of Pharmaceutical Education, 78(2), 36. https://doi.org/10.5688/ajpe78236

Proios, M. (2013). Athletic identity and social goal orientations as predictors of moral orientation. Ethics and Behavior, 23(5), 410-424. https://doi.org/10.1080/105084 22.2013.791622

Qaiser, Q. A., Iqbal, M., Abbasi, A., y Feroz, A. (2012). Level of moral development of first-year medical students: a study at a medical college in Pakistan. AJOB Primary Research, 3(2), 48-53. https://doi.org/10.1080/21507716.2011.652336

Quinlan, K. M. (2019). Emotion and moral purposes in higher education teaching: poetic case examples of teacher experiences. Studies in Higher Education, 44(9), 1662-1675. https://doi.org/10.1080/03075079.2018.1458829

Quinn, C., Kinnison, T., y May, S. A. (2012). Care and Justice orientations to moral decision making in veterinary students. Veterinary Record, 171(18), 4-46. https:// doi.org/10.1136/vr.101003

Rahnama, F., Mardani-Hamooleh, M., y Kouhnavard, M. (2017). Correlation between moral sensitivity and self-esteem in nursing personnel. Journal of Medical Ethics and History of Medicine, 10(16).

Ranjbar, H., Joolaee, S., Vedadhir, A., Abbasszadeh, A., y Bernstein, C. (2018). An evolutionary route for the moral development of nursing students: a constructivist grounded theory. Journal of Nursing Research, 26(3), 158-167. https://doi. org/10.1097/jnr.0000000000000224

Ranjbar, H., Joolaee, S., Vedadhir, A., Abbaszadeh, A., y Bernstein, C. (2017). Becoming a nurse as a moral journey: A constructivist grounded theory. Nursing Ethics, 24(5), 583-597. https://doi.org/10.1177/0969733015620940

Robles, V. (2013). La complementación de la discusión de dilemas con la lectura teórica para una eficaz intervención en el juicio moral. International Journal of Psychological Research, 6(1), 84-93.

Roche, C., y Thoma, S. (2017). Insights from the defining issues test on moral reasoning competencies development in community pharmacists. American Journal of Pharmaceutical Education, 81(8), 5913. https://doi.org/10.5688/ajpe5913

Rodríguez, L. M. (2016). Psicología del desarrollo moral en la adolescencia. Un modelo integrativo. Pontificia Universidad Católica Argentina. 
Rohling, M. (2017). Durkheim, Rawls e a educação moral. Revista Brasileira de Educaçâa, 22(71), 1-19. https://doi.org/10.1590/s1413-24782017227162

Romera, E. M., Ortega-Ruiz, R., Rodríguez-Barbero, S., y Falla, D. (2019). How do you think the victims of bullying feel? A study of moral emotions in primary school. Frontiers in Psychology, 10. https://doi.org/10.3389/fpsyg.2019.01753

Rzymska, I., Rzymski, P., Wilczak, M., Włoszczak-Szubzda, A., Jarosz, M. J., y Musielak, M. (2014). The influence of passive and active moral training on medical university on changes of students' moral competence index - Results from randomized single blinded trial. Annals of Agricultural and Environmental Medicine, 21(1), 161-166.

Sabatino, L., Rocco, G., Stievano, A., y Alvaro, R. (2015). Perceptions of Italian student nurses of the concept of professional respect during their clinical practice learning experience. Nurse Education in Practice, 15(4), 314-320. https://doi.org/10.1016/j. nepr.2014.09.002

Schmidt, C. D. (2014). Questioning intuition through reflective engagement. Journal of Moral Education, 43(4), 429-446. https://doi.org/10.1080/03057240.2014.951031

Schrepel, C., Jauregui, J., Brown, A., Shandro, J., y Strote, J. (2019). Navigating cognitive dissonance: a qualitative content analysis exploring medical students' experiences of moral distress in the emergency department. AEM Education and Training, 3(4), 331-339. https://doi.org/10.1002/aet2.10380

Seider, S., Novick, S., y Gomez, J. (2013). The effects of privileging moral or performance character development in urban adolescents. Journal of Early Adolescence, 33(6), 786-820. https://doi.org/10.1177/0272431612468318

Seiler, S., Fischer, A., y Voegtli, S. A. (2011). Developing moral decision-making competence: a quasi-experimental intervention study in the swiss armed forces. Ethics and Behavior, 21(6), 452-470. https://doi.org/10.1080/10508422.2011.622 177

Shek, D. T. L., y Zhu, X. (2019). Reciprocal relationships between moral competence and externalizing behavior in junior secondary students: a longitudinal study in hong kong. Frontiers in Psychology, 10, 528. https://doi.org/10.3389/fpsyg.2019.00528

Shields, D. L., Funk, C. D., y Bredemeier, B. L. (2018). Relationships among moral and contesting variables and prosocial and antisocial behavior in sport. Journal of Moral Education, 47(1), 17-33. https://doi.org/10.1080/03057240.2017.1350149

Skoe, E. E. A. (2010). The relationship between empathyrelated constructs and care-based moral development in young adulthood. Journal of Moral Education, 39(2), 191 211. https://doi.org/10.1080/03057241003754930 
Solum, E. M., Maluwa, V. M., Tveit, B., y Severinsson, E. (2016). Enhancing students' moral competence in practice: Challenges experienced by Malawian nurse teachers. Nursing Ethics, 23(6), 685-697. https://doi.org/10.1177/0969733015580811

Sorensen, D. P., Miller, S. E., y Cabe, K. L. (2017). Developing and measuring the impact of an accounting ethics course that is based on the moral philosophy of adam smith. Journal of Business Ethics, 140(1), 175-191. https://doi.org/10.1007/s10551-0152656-7

Šramová, B. (2017). Moral reasoning of adolescents. Turkish Online Journal of Educational Technology, 546-551.

Swisher, L. L. (2010). Moral reasoning among physical therapists: results of the defining issues test. Physiotherapy Research International, 15(2), 69-79. https://doi. org/10.1002/pri.482

Syabuddin, Jannah, M., y Sulaiman. (2020). The implementation of character education on the tarbiyah and teachers training faculty at the state Islamic University Indonesia (morality reinforcement approach). International Journal of Innovation, Creativity and Change, 12(12), 1-24.

Taş, H. (2019). Metaphors of elementary school students related to the lesson and teachers of religious culture and moral knowledge. Cumhuriyet Ilahiyat Dergisi, 23(1), 29-51. https://doi.org/10.18505/cuid.518013

Temli, Y., Sen, D., y Akar, H. (2013). Elementary school teacher candidates' perceptions and definitions on morality and moral education. Egitim ve Bilim, 35(168), 198214.

Tirri, K. (2011). Combining excellence and ethics: Implications for moral education for the gifted. Roeper Review, 33(1), 59-64. https://doi.org/10.1080/02783193.2011. 530207

Tomaschewisk-Barlem, J. G., Schallenberger, C. D., Ramos-Toescher, A. M., Barlem, E. L. D., Rocha, L. P., y Castanheira, J. S. (2020). Estratégias para o desenvolvimento da sensibilidade moral: perspectiva dos enfermeiros de unidades de terapia intensiva. Escola Anna Nery, 24(3), 1-7. https://doi.org/10.1590/2177-9465-ean-2019-0311

Torabizadeh, C., Homayuni, L., y Moattari, M. (2018). Impacts of Socratic questioning on moral reasoning of nursing students. Nursing Ethics, 25(2), 174-185. https://doi. org/10.1177/0969733016667775

Torres, A., Montilva, M., Ramírez, J., Zerpa, C., Zapata, E., García, M., Papale, J., Puertas, M., Franco, M., y Palencia, E. (2010). Desarrollo moral en estudiantes de medicina y enfermeria de la Universidad Centroccidental Lisandro Alvarado (UCLA) Venezuela. Revista de la Facultad de Medicina, 33(1), 36-41. http://ve.scielo.org/ scielo.php?script=sci_arttext \&pid=S0798-04692010000100007 
Trobec, I., y Starcic, A. I. (2015). Developing nursing ethical competences online versus in the traditional classroom. Nursing Ethics, 22(3), 352-366. https://doi. org/10.1177/0969733014533241

Tuvesson, H., y Lützén, K. (2017). Demographic factors associated with moral sensitivity among nursing students. Nursing Ethics, 24(7), 847-855. https://doi. org/10.1177/0969733015626602

UNESCO. (2020). Objetivos de Desarrollo Sostenible: Educación. UNESCO. https:// es.unesco.org/gem-report/node/1346

Valdés Cuervo, Á. A., Martínez, E. A. C., Quintana, J. T., y Madrid López, E. J. (2016). Relación entre funcionamiento familiar, emociones morales y violencia entre estudiantes de primaria. Revista Mexicana de Investigacion Educativa, 21(71), 10931110.

van Ommen, M., Daalmans, S., Weijers, A., Eden, A., de Leeuw, R. N. H., y Buijzen, M. (2017). A vigilante serial killer as ethics educator? An exploration of Dexter as a tool for moral education in the professional domain. Journal of Moral Education, 46(4), 378-395. https://doi.org/10.1080/03057240.2017.1338558

Van Stekelenburg, L. H., Smerecnik, C., Sanderse, W., y De Ruyter, D. J. (2020). 'What do you mean by ethical compass?' Bachelor students' ideas about being a moral professional. Empirical Research in Vocational Education and Training, 12(1). https:// doi.org/10.1186/s40461-020-00097-6

Vartiainen, T. (2010). Moral conflicts in project-based learning in ISD. Information Technology and People, 23(3), 265-280. https://doi.org/10.1108/09593841011069167

Vera-Estay, E., Dooley, J. J., y Beauchamp, M. H. (2015). Cognitive underpinnings of moral reasoning in adolescence: The contribution of executive functions. Journal of Moral Education, 44(1), 17-33. https://doi.org/10.1080/03057240.2014.986077

Verrinder, J. M., y Phillips, C. J. C. (2015). Assessing veterinary and animal science students' moral judgment development on animal ethics issues. Journal of Veterinary Medical Education, 42(3), 206-216. https://doi.org/10.3138/jvme.0215-022R

Vihos, J., Myrick, F., y Yonge, O. (2019). Socializing for authentic caring engagement in nursing practice: nursing student moral development in preceptorship. The Canadian Journal of Nursing Research, 51(2), 63-71. https://doi. org/10.1177/0844562118809258

Vílchez, M. P., Sánchez-Romero, E. I., Reche, C., y De Francisco, C. (2018). Moral development in people with intellectual disabilities through pre sport program . Revista Complutense de Educacion, 29(4), 1185-1199. https://doi.org/10.5209/ RCED.54953 
Walker, S., Brownlee, J., Whiteford, C., Cobb-Moore, C., Johansson, E., Ailwood, J., y Boulton-Lewis, G. (2012). Early years teachers epistemic beliefs and beliefs about childrens moral learning. Teachers and Teaching: Theory and Practice, 18(2), 263-275. https://doi.org/10.1080/13540602.2012.632267

Wang, C.-C., Chia, F., y Chang, C.-M. (2013). The study of college athletes' moral selfconcept and sport-related moral development. Life Science Journal, 10(4), 23612367.

Wiggleton, C., Petrusa, E., Loomis, K., Tarpley, J., Tarpley, M., O’Gorman, M. Lou, y Miller, B. (2010). Medical students' experiences of moral distress: development of a web-based survey. Academic Medicine : Journal of the Association of American Medical Colleges, 85(1), 111-117. https://doi.org/10.1097/ACM.0b013e3181c4782b

You, D., y Bebeau, M. J. (2012). Gender difference in ethical abilities of dental students. Journal of Dental Education, 76(9), 1137-1149.

Yuguero, O., Esquerda, M., Viñas, J., Soler-Gonzalez, J., y Pifarré, J. (2019). Ethics and empathy: The relationship between moral reasoning, ethical sensitivity and empathy in medical students . Revista Clinica Espanola, 219(2), 73-78. https://doi. org/10.1016/j.rce.2018.09.002

Zerpa, C. (2007). Tres teorías del desarrollo del juicio moral: Kohlberg, Rest, Lind. Implicaciones para la formación moral. Laurus, 13(23), 137-157. https://www. redalyc.org/articulo.oa?id=76102308

Zhao, Y. P., Xia, Y. W., Chen, X., y Miao, X. H. (2018). Ideological education of college students in China: Based on the distribution characteristics of moral development stage. Kuram ve Uygulamada Egitim Bilimleri, 18(5), 2571-2581. https://doi. org/10.12738/estp.2018.5.160

Recibido: 23 de marzo de 2021

Aceptado: 18 de mayo de 2021 